\title{
Interactive comment on "Modeling long-term fire impact on ecosystem characteristics and surface energy using a process-based vegetation-fire model SSiB4/TRIFFID-Fire v1.0" by Huilin Huang
} et al.

Huilin Huang et al.

hhllbao@ucla.edu

Received and published: 19 September 2020

The comment was uploaded in the form of a supplement:

https://gmd.copernicus.org/preprints/gmd-2020-122/gmd-2020-122-AC2-

supplement.pdf 